\title{
Context Aware Healthcare Application
}

\author{
Shankari Bhattacharyya \\ M.Tech Computer Science \\ School of Computing Science \\ and Engineering, VIT University, \\ Vellore-632014, Tamil Nadu
}

\author{
RA.K.Saravanagru \\ Assistant Professor Senior \\ School of Computing Science \\ and Engineering, VIT University, \\ Vellore-632014, Tamil Nadu
}

\author{
Arunkumar Thangavelu \\ Professor \\ School of Computing Science \\ and Engineering, VIT University, \\ Vellore-632014, Tamil Nadu
}

\begin{abstract}
Context-awareness is considered as an enabling technology and a rich area of application. This paper aims in monitoring and reporting critical health conditions of a patient who is suffering from brain tumor. We have built our ontology starting from knowledge gathering of data and reasoning to identify the health condition of the patient. So this has led to move towards to build an abstract framework for context-aware applications.
\end{abstract}

\section{Keywords}

Context-aware, health care, ontology, patient monitoring, rulebased systems.

\section{INTRODUCTION}

The history of artificial intelligence shows that knowledge is the most critical for intelligent systems. Better knowledge is very important for solving any tasks. In order to have a truly intelligent system, knowledge needs to be captured and then processed and lastly it should be well communicated. All the above tasks are supported by Ontologies. Ontologies capture knowledge about some domain of interest and also describe the relationship that hold between those concepts. All around the world, the population is increasing, putting an enormous pressure on health systems. It is very difficult to predict or assume what the future hospital will be. The relevant physiological data, several variables related to the environment can be collected and interpreted in real-time. Context-Aware system considers this kind of information in a comprehensive fashion and improves the quality of medical care. In this paper we discuss about the possible solution and the various challenges and difficulties faced in context awareness in health care.

The various uses of computerized tools such as powerful hospital information system is increasing in day to day life but these tools are not enough and the new technologies should support the new way of visualizing the future hospitals. So developing the future tools will require new technologies and also new architectures in order to develop secure and reliable system. The main focus is on indentifying, implementing new services for communication and cooperation of health care professionals. By communication we mean that it involves larger activities may be direct or indirect like laboratory results to consultations and advices. In almost all hospitals the doctors do take the utmost care of patients but the breakdown in communication causes error and which may lead to the death of the patients.
Thus cooperation between health care professionals can be done trough computerized platforms say home care coordination for elder patients who will integrate new mobile tools and also proposes new communication abilities. Context-awareness is a concept and the technologies such as sensors, wearable instruments, intelligent artifacts, handheld computers etc. are available for the development of the new application which will make the health care professionals to manage their tasks and also it will increase the quality of the patient care.

The research presented in this thesis is about how the inference is done mainly on knowledge base in an easier manner as compared to the rule based approach. The Protégé help us in designing the ontology for the medical domain and how the reasoner works and helps us inferring the correct suggestion in diagnosing the disease with the symptoms available in the knowledgebase.

\section{INTRODCTION TO CONTEXT AWARE AND CONTEXT AWARE COMPUTING}

Schilit and Theimer (1994) refer to context as location, identities of nearby people and objects, and changes to those objects. Brown, Bovey et al. (1997) [13] define context as location, identities of the people around the user, the time of day, season, temperature, etc. Dey and Abowd's definition (2001) [12]: Context is any information that can be used to characterize the situation of an entity. "An application ability to adapt to changing circumstances and respond according to the context of use" [11] is defined as context aware computing. According to the definition of author Dey and Abowd's definition (2001) [12] context-aware is if it uses context to provide relevant information and / or services to the user where the relevancy depends on the user's task.

\section{RELATED WORK}

Some of the existing context aware medical applications are: In VOCERA COMMUNICATION SYSTEM [2] experimentation was done in St. Vincent Hospital, Birmingham, USA. Before in St. Vincent Hospital had 802.11 networking to support mobile clinical users of wireless PDAs and laptops. STROKE ANGEL [3] experiment was conducted at Bad Neustadt, Germany. (November 2005-May 2008). The main aim of this system is to shorten the time requirement for the entire process. That is from discovery and diagnosing the stroke victim to the patient's admission and treatment in hospitals. This is the main goal is to meet the short time frame in which to treat the stroke patient the first 3 hours after the stroke. In INTELLIGENT HOSPITAL 
SOFTWARE, UNIVERSITY OF CAMBRIDGE,UK [4] an experimental prototype has been implemented after the study of needs at the Accident \& Emergency Department of the Royal London Hospital. One more study was conducted for a period of 8 months in a mid size public hospitals in the city of Ensenada, Mexico [5]. The emphasis was done on the activities performed by three roles. They are Nurses, Physicians and the medical interns A decision-level data fusion technique for monitoring and reporting critical health conditions of hypertensive patients [6].Three relevant classes of information have been identified to gather through pervasive device. These are: Environmental, Psychological, and Behavioral. The issues discussed in this paper are: individualization of treatment, influence of the patient activity and of the home environment, relaxation (or loosening) of the limits of each monitored variable, Based on the issues they have proposed some architectural framework - HSAUDE (Health Support in Aware and Ubiquitous Domestic Environments).

\section{CONTEXT AWARE PATIENT MONITORING SYSTEM}

\subsection{The Challenges:}

The escalating worldwide growth in the elderly population, chronic conditions and the costs associated with caring for them necessitates a new model for care and collaboration. This application will provide a standard way for healthcare providers to exploit new sources of data from medical devices and sensors for smarter healthcare. So the solution is the use of sensorenabled medical devices to collect vital data such as blood pressure, weight, glucose level and automatically transmit the results to a personal health system, then to the monitoring service .The care team can keep a close track on the patient's health condition.

\subsection{The Benefits:}

It will monitor vital health related data. Data from sensors and medical devices located within a patient's home, combined with application can deliver new insights to improve healthcare management across three key domains:

- Chronic disease management - case study on brain tumor

- $\quad$ Elderly monitoring (future enhancement)

- Health and wellness is the assessment on the health conditions based on the monitoring parameters.

\section{SYSTEM ARCHITECTURE}

The following system architecture has been followed (Figure:1). It has three modules: Context Management, Reasoning and Notification.

\subsection{Context Management:}

The primary and the most important mechanism and refinement of data are contained in context management. It also performs data filtering and helps in extracting the relevant features. Inference is used to generate new information which may be useful for reasoning. This module also interacts with the devices when the values changes for any sensors.

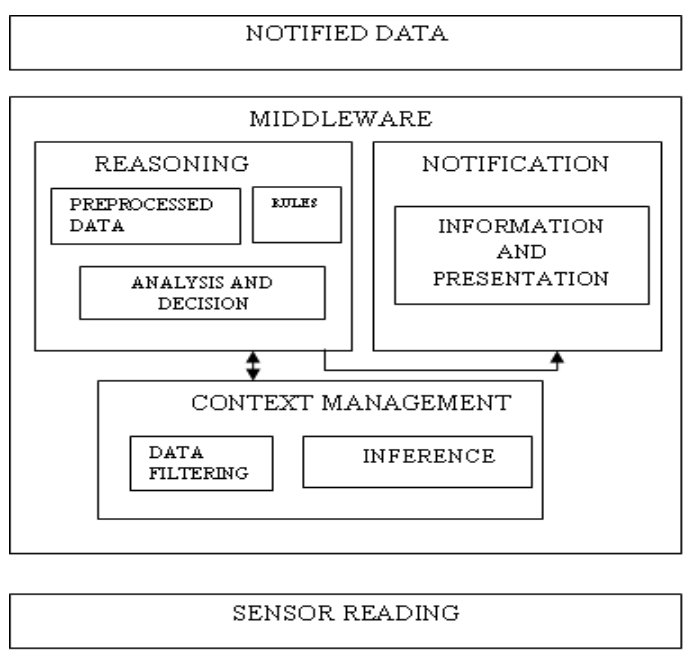

Figure 1: System Architecture

To structure the concept of context we have used following concept [9]:

- A context describes a situation and the environment a device or user is in.

- Unique name is used to identify a context.

- A set of relevant features is related to each context

- And for each relevant feature a range of values can be determined (say both implicitly or explicitly) by the context.

Based on the above concept an organized feature space for context can be developed. At the top level we have context related to human factors, and context related to the physical environment. And then we go ahead with further classification.

Human factors related context is divided mainly into two categories: information on the user which includes knowledge of habits, emotional state, physiological conditions, etc., and the user's activities like spontaneous activity, engaged tasks, or idle sate etc. Similarly, context related to physical environment is structured into two categories: location which may include absolute position, relative position, etc. and physical conditions like light, pressure, heart rate, temperature etc. In each category, relevant features can be identified, whose values will help in determining context. (Figure: 2).

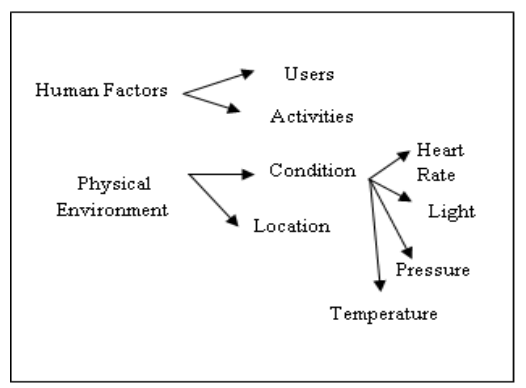

Figure 2: Context Feature Space. 
Authors like Albrecht Schmidt, Michael Beigl, and Hans-W. Gellersen [9] said that context can be acquired from either explicitly by requiring the users to specify it or implicitly by monitoring the user. So in our application we have a set of basic symptoms that are related to brain tumor and the based on the patient response the diagnosis is carried out.

System can use pre-defined rules written in some logic forms to deduce different contexts. For example, based on the number of symptoms of brain tumor turns to be positive, the system can deduce some conclusions. It uses rules to perform the deduction. Some of the rules used in our applications are:

1. \#patient(Symptom" $>=$ "16 AND Symptom " $<=$ "20 ) $>$ Patient (chances brain tumor, second level checkup).

2. \#patient (Symptom "<"4) $=>$ Patient (Normal, check up if needed).

Architecture of sensor fusion [9] is a layered architecture for context awareness with sensors, cues, context and scripting layers. For sensors we have two kinds of sensors namely the physical and the logical sensors. All the sensors return a scalar, vector or symbolic value and they are time dependent. Taking the values from a single sensor for a certain time interval and then providing a symbolic output is known as cue. Each cue is dependent on a certain sensor but different cues may be based on the same sensor. A context is a description of the current situation that is based on cues and lastly the scripting layer provides mechanism to include the context information in an application. First is entering a context: It means that when a certain situation is noted with a higher probability, which is greater than the threshold value, an action is performed after a certain time. If patient is reporting a seizure which he never had before then he must do a MRI scan for the brain. Here the situation is seizure and the probability lies in the higher range as he had never suffered before so the action that is the MRI scan is performed. Secondly while in a context: It means that when a certain situation is noted with a higher probability, which is greater than the threshold value an action is performed every specified time interval. If the patient is suffering from severe headache then it must be taken care about at what particular time or in which situation the headache is more. And lastly leaving a context: If the probability for a certain situation is becoming less than the threshold value an action will be performed at a certain time.

\subsection{Reasoning:}

This is another important module as based on the analysis it will generate an alarm. It will receive all the preprocessed data from the various sensors as input values and then it will analyze and determine the critical situation which may need an emergency alarm. The decision will be taken based on the rules which are configured made from the various variables and these rules are used by the decision component according to the current context. For example, in the case of blood pressure and heart rate monitoring system, the following environmental, physiological and behavioral variables rules are set:

- If the average systolic pressure is greater than $135 \mathrm{mmHg}$ and the diastolic are greater than $85 \mathrm{mmHg}$ then the patient is considered to be having hypertensive [7].

- If the patient is eating, the systolic pressure should raise not more than $8.8 \mathrm{mmHg}$ above the average and the diastolic to $9.6 \mathrm{mmHg}$ [8].

Even for deciding the severity of brain tumor check-ups are performed and results are scaled between 1 to 20.Depending on the results, next tests are performed.

The most basic way to preprocess a data stream from sensor is by using statistical methods [10].These values are known as features or cues as they denote a stream of data by extracting a single value. Let's say from the temperature sensor the following features are noted maximal and minimal temperature and the average temperature etc.

Context refers to some information that describes some aspect of conditions in which an application will execute. The feature extraction layer (FE) [10] concepts can be used and this strictly separates the sensor layer and the context consuming layer. In this FE we have feature tuple space (FT) which supports the interoperability between heterogeneous sensor nodes. It is represented as:

FT $=\{$ Sensor_type_id, feature_id, feature_value, timestamp $\}$

With the help of this we have derived some primitive context directly say when the temperature sensor measures and finds its above or the blood pressure monitoring system gives a reading which falls above the range normal then it can provide notification to the care person to give the basic medical help Context data is derived from various available features. The various methods for generating context from primitive features can be rule-based algorithms, statistical method or reasoning machine [10]. In our application we have used the reasoning techniques where the domain knowledge is gathered and the knowledge base is made using ontology which helps in diagnosing. It helps in the process of context acquisition and the context tuple space can be written as:

$\mathrm{CT}=\{$ Context_id, attribute_id, context date, time, probability $\}$, where the attribute is having an id, date and time adds additional information about the context and about other contextual information.

We follow a bottom-up approach where we collect the data from the various hardware sensors. It extracts features form various heterogeneous sensors and then deriving context information from those features and then deliver it to the application that are running. We also enter some values manually and then generalize the value based on the context reasoning mechanism.

\subsection{Notification:}

The system will generate an alarm which will be indicated when there is a deviation from the expected medical recommendations and emergencies which may require an urgent medical assistance. For example a patient is suffering from malignant tumor. He has come for re-checkup as these types of tumor have chances of re-growth. After checkup a comparative analysis would be done to find if there is a deviation in results. If the 
difference is critical, an alarm will triggered to doctor whom the patient is reporting to.

\section{IMPLEMENTATION}

This application mainly helps in monitoring the patient who is diagnosed with brain tumor. The patient has already been registered and undergone treatment. All his data will be recorded and maintained on the next visit of the patient this application will retrieve the record with the help of the patient's identification number which will help in diagnosing by comparing the previous test results with the latest. Lastly a report will be generated with all the medical treatment details which the patient has undergone.

Implementation consists of two parts. First part contains patient monitoring system, in which ASP C\#.NET has been used as the front end. Front end take cares of Patient Monitoring basic checkup, first level of checkup, second level of check up and the final checkup. And we have used SQL SERVER 2005 as backend database. It stores information about Doctor, Patient and all levels of checkup information. The second part consist of building up and OWL ontology with existing facts and to inferred some new facts from the existing facts. Here we have used Protégé 4.0 beta to create our OWL ontology. Through Protégé 4.0 OWL editor we have created classes, data and object properties and individuals (core part of OWL ontology). This OWL ontology will serve as a knowledge base for our application. With OWL API version 2.0 we have manipulated Patient.owl ontology.

If a new patient arrives he has to register with full details before undergoing the checkups.Details like patient's name, his father/husband /guardian name, gender, address,blood group,weight, height, contact number, nationality needs to be entered.For every new patient an unique id is generated. Patient would then continue with the next levels for check-ups. Simailarly a doctor registration details is also maintained which may be useful for getting the information about a particular doctor later. The patient diagnosis is mainly done in four levels. Firstly, the primary checkup level where some of the readings are based on the context which are taken(from sensors) and some values are entered manually The description of the various data collected are from pulse oximetry which is a noninvasive method allowing the monitoring oxygenation of a patient's haemoglobin. Sphygmomanometer- an instrument used for measuring blood pressure. Glucometer which is also known as glucose meter. It is a medical device used for determining the approximate the concentration of glucose in the blood and various other instruements..Medical meter readings would then check with the knowledge base which is working on a narrow domain to provide intelligent decisions with justification. Knowledge base contains a set of data in the form of rules that describe the knowledge in a logically consistent manner.Knowledge is acquired and represented - if the values are normal or is within the range and based on the inference it displays the result.

The reasoner is able to reason out or inferred the new facts from the existing facts with the help of Hermit reasoner (there are other reasoners too like Fact++ and Pellete 1.5 added in OWL API Version 3.0). Lastly by using
Netbeans 6.9.1 we have manipulated OWL Ontologies as OWL API's are written in java.

\section{RESULTS AND DISCUSSION}

The screenshot below is the basic checkup that is done to a patient. On the left hand side shows the analysis of the data captured. (Figure 3).

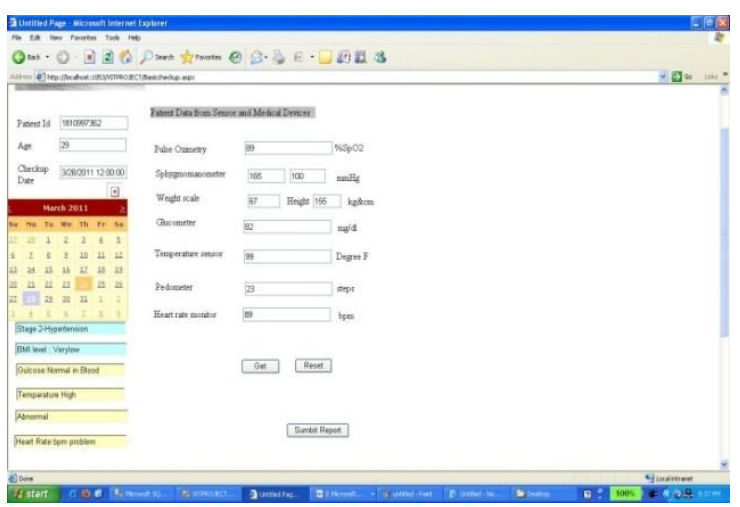

Figure 3: Pre check up module

For example The American Heart Association has recommended some guidelines to define normal and high blood pressure.

Nornal blood pressure : 120/80 $\mathrm{mmHg}$.

Prehypertension: 120-139/80-89 mmHg.

High blood pressure (stage I): 140-159/90-99 mmHg.

High blood pressure (stage II): higher than 160/100.

Similarly the normal or standard value for heart rate monitor is $60-80 \mathrm{bpm}$. The mean nornal blood glucose level in human is about $4 \mathrm{mg} / \mathrm{dl}$. And the acceptable normal ranges for pulse oximetry are from $95 \%-100 \%$ although values down $90 \%$ are common.

The next level of check up analyzes the basic symptoms of brain tumor. The inference engine tries to derive information from the knowledge base based on the answers - the critivality of the disease with an indication if the patient needs to go for the second level of checkup. Some of the common symptoms that are related to barin tumor is whether the patient is having headache with addition to fever and whether he is feeling unconscious, whether pain is there in neck or back or while stressing limbs We have taken the symptom result on a scale of 1 to 20 . The depiction is as follows:

- If result $>=16$ and result $<=20$ then it will display a message " He has a chance for $40 \%$ of brain tumor so need to go for a second level of treatment"

- If result $<16$ then he have $25 \%$ of symptoms only.

In the second stage of checkup the patient will be checked with more detailed symptoms of brain tumor. Some of the symptoms which clsoely related to brain tumor are if the patient completely loses his vision, or facing completely hearing loss or the he is having difficulty in speaking or the speech delivered by him is inappropriate. We have scaled it between 1 to 18 . The depiction are : 
- If result is $>=16$ and result $<=18$ then the inference is "Very sick: active supportive care needed and have to go for a MRI scan."

- If the result is $<16$ then the patient requires assistance care and should remain under restrictions.

Figure 4 vshows the complete report of a particular patient.

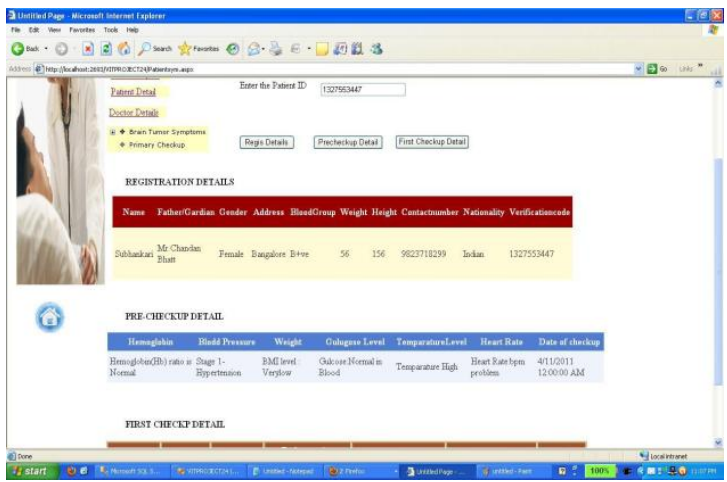

Figure 4: Full Report of a particular patient

In Figure 5: It shows the knowledge base created in Protégé 4.0 to create our Patient OWL ontology. Our ontology consists of Disease, Symptoms, Patient and Severity as main classes. Disease class contains several subclass of disease or direct information about particular disease (like brain tumor as you can see from the figure). Symptoms class contains all possible symptoms of several diseases. And patient class contains information about particular patient like Age, Gender , Symptoms etc. Several data and object property to create restriction on classes that will serve as necessary and sufficient condition for several classes.

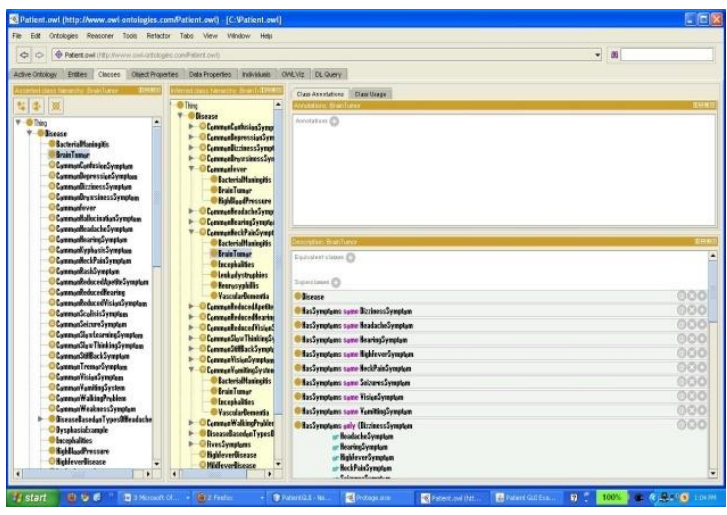

Figure 5: Patient.owl Ontology

We have created several defined classes that contain necessary and sufficient condition for the disease. In Figure 6 it shows that if we select particular class and click on "classify", then the OWL API 2.0 will reason out the sub classes of defined classes. We have used OWL Reasoner that intern uses Hermit reasoner.

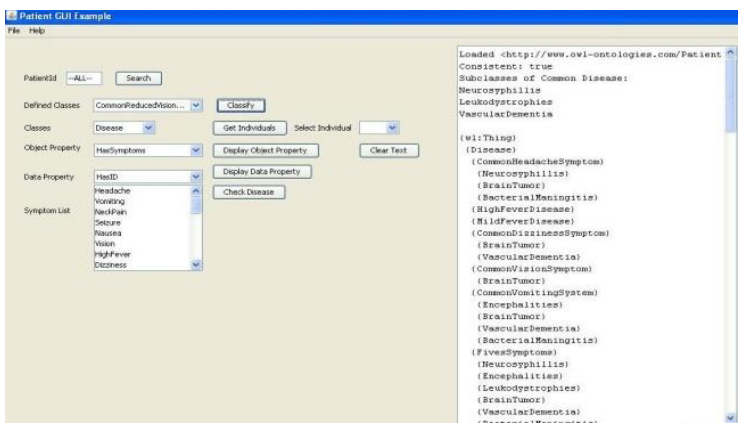

Figure 6: Patient GUI showing Classification

In Figure 7 based on the user symptom we can select particular symptom and application will give you the list of disease the patient might have. The inference is done form the knowledge base that we have already created.

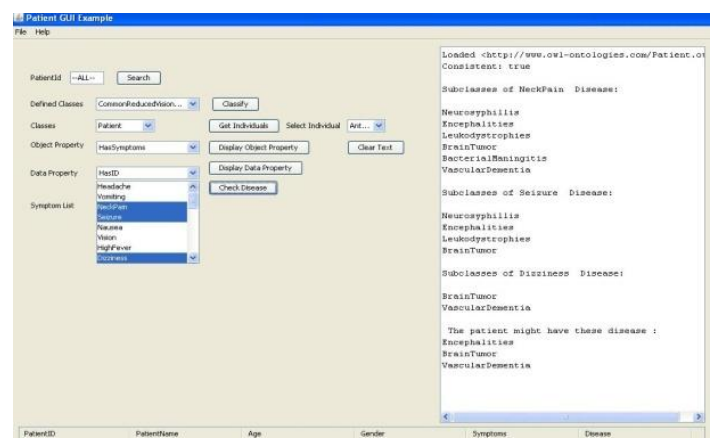

Figure 7: Patient GUI showing Inference of Disease

\section{CONCLUSION AND FUTURE WORKS}

Large amount of various information are required for health care application. Many researchers have considered and discussed the importance of location as an important attribute in context aware systems. It is apparent to the authors that developing a context aware application is not a problem to access to technologies say sensors, networks etc. But the difficulty is that the research communities have not reached to any agreement in deciding the best way to model context and architecture to support its use. The development of such system should have expertise from various disciplines including computer science, design and evolution, social etc.

As for future enhancement if an elderly individual who is being monitored falls or forgets take their pills, the person who is taking care should be notified to help the patient. In addition to this for elderly individuals who want to continue living independently, but need some monitoring to ensure they are safe, system can allow caregivers to use sensors to assist with daily health and monitoring tasks, and provide early warning using sensor data collection 


\section{ACKNOWLEDGMENTS}

Our sincere thanks to Dr. G.Viswanathan, Chancellor, VIT University, Vellore, for such infrastructure and support provided and also we are indebted to Dr. M.Khalid, Director, School of Computer Science and Engineering, VIT University, Vellore for his immense support.

\section{REFERENCES}

[1] Nathalie Bricon-Souf, Conrad R. Newman: "Context Awareness in Health Care a Review:" , International Journal of Medical Informatics 76(1), 2-12, 2007.

[2] Vince Stanford: "Beam me up, Dr. McCoy", IEEE Pervasive Computing Magazine, pp. 13-18, 2(3) , 2003.

[3] Carsten Orwat, Asarnusch Rashid, Carsten Holtmann, Michaela Wolk, Mandy Scheermesser, Hannah Kosow, Andreas Graefe "Adopting Pervasive Computing for Routine Use in Healthcare" , IEEE Pervasive Computing:(vol. 9 no. 2) , pp.64-71, April-June 2010.

[4] Scott Mitchell, Mark, D.S., John Bates, George Coulouris "Context-aware multimedia computing in the intelligent hospital", ACM SIGOPS European Workshop Proceedings of the 9th workshop on ACM SIGOPS European workshop: beyond the PC: new challenges for the operating system, pp. 13-18, 2000.

[5] Jesus Favela, Monica Tentori, Luis, A.C., Victor M.G., Elisa B.M., Ana I.M.G "Estimating Hospital Work Activities in Context-Aware Healthcare Applications", Mexico City Pervasive Health Conference and Workshops, pp.1-10, 2006.

[6] Alessandro Copetti, Orlando Loques, Leite, J.C. B., Thais P. C.Barbosa, da Nobrega, Antonio C.L. "Intelligent contextaware monitoring of hypertensive patients" , Pervasive Technologies for healthcare,2009 .Pervasive Health 2009. 1-3, pp.1-6, April 2009.

[7] Mogi das Cruzes, Sao Paulo "IV Guideline for Ambulatory Blood Pressure Monitoring. II Guideline for Home Blood Pressure Monitoring", Arquivos Brasileiros de Cardiolologia, vol. 85 (Supl11), pp.1-18, 2005.

[8] D. Mion, W. Oigman, and F. Nobre "MAPA: Monitorizacao Ambulatorial da Pressao Arterial", Atheneu RJ, 3rd ed., 2004.

[9] Albrecht Schmidt, Michael Beigl, Hans-W. Gellersen, "There is more to context than location", Computers and Graphics, vol. 23, No. 6, pp.893-901, 1999.
[10] N.Q.Hung, L.X.Hung and S.Y. Lee. "A Middleware Framework for Context Acquisition in Ubiquitous Computing Systems.", Second International Conference on Computer Applications (ICCA 2004), Myanmar, 8th, January, 2004.

[11] J. Kjeldskov, M. Skov "Supporting work activities in healthcare by mobile electronic patient records," in: Proceedings of the 6th Asia-Pacific Conference on Human-Computer Interaction, APCHI 2004, Rotorva, New Zealand,2004.

[12] Anind K.Dey, Gregory D.Abowd "Toward a better understanding of context and context-awareness.", GVU Technical Report GIT-GVU-99-22, College of Computing, Georgia Institute of Technology, 1999

[13] Matthias Baldauf, Schahram Dustdar, Florian Rosenberg, "A survey on context-aware system." International Journal of Ad Hoc and Ubiquitous Computing, Volume 2 Issue 4, June 2007.

\section{AUTHORS PROFILE}

Shankari Bhattacharyya is currently pursing Master of Technology in Computer Science and Engineering (20092011) from Vellore Institute of Technology, Vellore, Tamil Nadu. Her research interest includes Context Aware system, Web services and Ontology.

RA.K. Saravanaguru is working as Assistant Professor Senior in School of Computer Science \& Engineering at VIT University. His research work focuses on Context-Aware Systems, Vehicular Ad hoc Networks, Middleware Technologies, Semantic Web and Web Services.

Arunkumar Thangavelu is currently associated with Vellore Institute of Technology University, India. He has over 16 years of academic, Research experience in field of adhoc, sensor networking, DataMining. He is an active consultant in $\mathrm{R} \& \mathrm{D}$ planning, proposal evaluation as well reviewer in international / national journals. He had received his Doctoral Degree from PSG College of Technology. His domain of research expertise varies from mobile / vehicular adhoc networks, distributed computing to precision agriculture. 